\title{
Concurrent use of nivolumab and radiotherapy for patients with metastatic non-small cell lung cancer and renal cell carcinoma with oligometastatic disease progression on nivolumab
}

\author{
JAWAHER ANSARI $^{1,2}$, ASHRAF FARRAG $^{1,3}$, ARWA ALI $^{1,4}$, MAI ABDELGELIL $^{1,3}$, ESAM MURSHID $^{1}$,

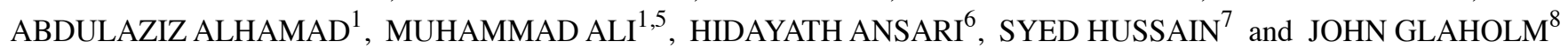 \\ ${ }^{1}$ Department of Oncology, Prince Sultan Military Medical City, Riyadh 12233, Saudi Arabia; ${ }^{2}$ Department of Oncology, \\ Tawam Hospital, Al Ain 15258, United Arab Emirates; ${ }^{3}$ Clinical Oncology Department, Assiut University Hospital; \\ ${ }^{4}$ Medical Oncology Department, South Egypt Cancer Institute, Assiut University, Assiut 71515, Egypt; \\ ${ }^{5}$ Department of Radiation Oncology, Icon Cancer Centre, Warrnambool, Victoria 3280, Australia; ${ }^{6}$ Department of Imaging, \\ Cleveland Clinic Abu Dhabi, Abu Dhabi 112412, United Arab Emirates; ${ }^{7}$ Department of Oncology and Metabolism, \\ University of Sheffield, Sheffield S10 2RX; ${ }^{8}$ Department of Oncology, Royal Marsden Hospital, London SW3 6JJ, UK
}

Received October 3, 2020; Accepted July 9, 2021

DOI: $10.3892 / \mathrm{mco} .2021 .2376$

\begin{abstract}
Checkpoint inhibitors (CPIs), such as nivolumab, have transformed the treatment paradigm for patients with metastatic non-small cell lung cancer (mNSCLC) and metastatic renal cell carcinoma (mRCC). The combination of CPIs and radiotherapy (RT) constitutes a multimodal treatment approach that may work synergistically and facilitate augmented systemic responses. The aim of the present retrospective study was to assess the efficacy and safety of continuation of nivolumab treatment with the addition of RT in patients with mNSCLC and mRCC who develop oligometastatic disease progression on single-agent nivolumab. All patients with $\mathrm{mNSCLC}$ and $\mathrm{mRCC}$ who received nivolumab at the Department of Oncology, Prince Sultan Military Medical City (Riyadh, Saudi Arabia) between November 2016 and April 2018 were identified. The records of patients who developed oligometastatic disease progression during nivolumab treatment and were subsequently treated with RT, with nivolumab continued beyond disease progression, were retrospectively reviewed. Details of RT, clinical outcomes and toxicity data were collected. Of the 96 patients who received nivolumab, 22 received multiple courses of RT. A total of 39 sites were irradiated: Bone $(n=15)$, lung $(n=9)$, brain $(n=8)$, adrenal gland $(n=2)$, renal bed $(n=2)$, skin $(n=1)$, ethmoid sinus $(n=1)$ and scalp $(n=1)$. Partial response and complete
\end{abstract}

Correspondence to: Dr Jawaher Ansari, Department of Oncology, Tawam Hospital, Maqam Street, Al Maqam, P.O. Box 15258, Al Ain 15258, United Arab Emirates

E-mail: jak.ansari@gmail.com

Key words: nivolumab, renal cell carcinoma, lung cancer, concurrent, radiotherapy, stereotactic body radiotherapy, checkpoint inhibitors, pseudoprogression response were noted at $25(64 \%)$ and $3(8 \%)$ sites, respectively. Stable disease was noted at 6 sites $(15 \%)$ and disease progression was noted at 5 sites (13\%). The median time on nivolumab from the date of the first fraction of RT was 4.5 months (range, 1.5-29 months) for patients with mNSCLC and 5 months (range, 1-38.5 months) for patients with mRCC. No patients developed grade 3-4 toxicities. Grade 2 pneumonitis was noted in 3 patients receiving lung RT. The addition of RT appeared to initiate a response and prolong the duration of nivolumab treatment. Therefore, the combination of nivolumab and RT was found to be well tolerated, with response rates exceeding those in published studies of nivolumab monotherapy.

\section{Introduction}

Recent advances in immunotherapy, particularly checkpoint inhibitors (CPIs), have transformed the treatment paradigm for patients with metastatic non-small cell lung cancer (mNSCLC) and metastatic renal cell carcinoma (mRCC). Nivolumab is a human immunoglobulin G4 monoclonal antibody, which binds to the programmed death-1 (PD-1) receptor and blocks its interaction with its ligands, programmed death-ligand (PD-L) 1 and PD-L2, potentiating T-cell responses (1). The United States Food and Drug Administration (FDA) initially approved nivolumab monotherapy in 2015 for patients with $m$ NSCLC who develop progressive disease (PD) on or after platinum-based chemotherapy. In 2020, nivolumab received FDA approval as first-line treatment of patients with mNSCLC (without EGFR or anaplastic lymphoma kinase genomic aberrations) in combination with ipilimumab, an anti-cytotoxic T-lymphocyte antigen-4 antibody, for patients expressing PD-L1 $>1 \%$; or for all comers, regardless of PD-L1 expression, in combination with two cycles of platinum doublet chemotherapy. For patients with mRCC, nivolumab has been FDA-approved in the second-line setting after prior anti-angiogenic therapy and in treatment-naive intermediate- to poor-risk patients in combination with ipilimumab. 
Nivolumab is effective and well-tolerated in patients with mNSCLC and $\mathrm{mRCC}$; however, the response rates with monotherapy remain low $(\sim 20 \%)$ and most patients eventually develop PD. Therefore, immunotherapy trials are currently focusing on immunotherapy combinations to enhance therapeutic efficacy. Recent clinical trials have shown improvement in clinical outcomes by combining CPIs with other CPIs or chemotherapeutic agents $(2,3)$. However, the clinical application of these combinations may be limited by prohibitive cost and overlapping additional toxicities.

In the phase III CheckMate 067 trial for patients with advanced melanoma, the combined administration of nivolumab and ipilimumab demonstrated improvement in progression-free survival (PFS) and overall survival (OS) when compared to monotherapy with either drug alone; however, the combination was associated with grade 3-4 toxicities in $59 \%$ of the patients (3). CheckMate 214, a phase III trial for patients with $\mathrm{mRCC}$, demonstrated improved OS for patients with intermediate- to poor-risk mRCC when compared to sunitinib. However, the combination was associated with grade 3-4 toxicities in $46 \%$ of the patients and toxicity-related treatment discontinuation in $22 \%$ of the patients.

Radiotherapy (RT) is an important cancer treatment modality, which is indicated in $\sim 52 \%$ of all patients with cancer (4). RT has limited side effects and is highly cost-effective, accounting for only $5 \%$ of the total cost of cancer care, making it an attractive modality for using in combination with other systemic therapies (5). RT may augment the immune response achieved with the use of CPIs, either through direct cytotoxic antitumour effect or by modifying the tumour microenvironment to promote an antitumour immune response (6). The antitumour effects of RT have also been observed outside the radiation field, referred to as the 'abscopal effect' (7).

The aim of the present retrospective study was to assess the safety and efficacy of nivolumab and concurrent RT for patients with $\mathrm{mNSCLC}$ and $\mathrm{mRCC}$ with oligometastatic PD on nivolumab monotherapy.

\section{Patients and methods}

Patients. A total of consecutive 96 patients who received nivolumab at the Department of Oncology, Prince Sultan Military Medical City (Riyadh, Saudi Arabia) between November 2016 and April 2018 were retrospectively identified. The inclusion criteria for this study were patients who developed oligometastatic PD during nivolumab treatment and were subsequently treated with RT, with nivolumab continued beyond disease progression. A total of 22 patients met the criteria, of whom 12 had mNSCLC and 10 had mRCC. For the study population, a comprehensive chart review was completed. Clinical data and RT details were collected from our electronic medical records, as well as the RT planning system (ARIA OIS; Varian Medical Systems, Inc.). Patient demographic data and radiological and pathological details were obtained, in addition to RT indications, treatment sites, techniques and fractionations.

Treatment. Nivolumab treatment was administered intravenously as per the standard protocol (https://www.accessdata. fda.gov/drugsatfda_docs/label/2021/125554s090lbl.pdf), at a dose of either $240 \mathrm{mg}$ every 2 weeks or $480 \mathrm{mg}$ every 4 weeks. All patients were assessed by the clinician prior to each nivolumab administration.

RT was delivered as per standard departmental protocols for stereotactic or conformal treatment. RT was administered with a linear accelerator and delivered as conformal RT, or intensity-modulated RT, as clinically appropriate. Stereotactic body RT (SBRT) was delivered with linear accelerator whereas Gamma Knife (Elekta Instrument AB) was used for stereotactic radiosurgery (SRS). Fractionated RT was generally administered as a palliative hypofractionation schedule determined according to the discretion of treating physician. The fractionation schedules for conformal external beam RT included $20 \mathrm{~Gy} / 5$ fractions (fr), $30 \mathrm{~Gy} / 10 \mathrm{fr}, 25 \mathrm{~Gy} / 5 \mathrm{fr}$, $20 \mathrm{~Gy} / 4 \mathrm{fr}, 16 \mathrm{~Gy} / 4 \mathrm{fr}$ and $8 \mathrm{~Gy} / 1 \mathrm{fr}$. The fractionation schedules for stereotactic RT included $48 \mathrm{~Gy} / 4 \mathrm{fr}, 40 \mathrm{~Gy} / 10 \mathrm{fr}, 40 \mathrm{~Gy} / 4 \mathrm{fr}$, $34 \mathrm{~Gy} / 4$ fr and $22 \mathrm{~Gy} / 1 \mathrm{fr}$.

Response assessment. Objective clinical response [complete response (CR), partial response (PR), stable disease (SD) or $\mathrm{PD}]$ was evaluated using CT, MRI or ${ }^{18} \mathrm{~F}$-fluorodeoxyglucose positron emission tomography-CT scans.

The modified Response Evaluation Criteria In Solid Tumours criteria, version 1.1, were adopted for the assessment of response at target lesions receiving RT (8). Response was defined as follows: CR, complete disappearance of the target lesion; PR, at least $30 \%$ decrease in the longest diameter of the target lesion; $\mathrm{PD},>20 \%$ increase in the longest diameter of the target lesion; and SD, neither sufficient shrinkage to qualify for PR, nor sufficient increase to qualify for PD. Patients considered as $\mathrm{CR}, \mathrm{PR}$ or $\mathrm{SD}$ should not have unequivocal PD in the non-target lesions. Adverse events and laboratory abnormalities were evaluated using the National Cancer Institute Common Toxicity Criteria (version 3.0) of the National Cancer Institute (9).

\section{Results}

Patient characteristics and adverse events. A total of 22 patients received multiple courses of RT. The median age of the patients was 59 years (range, 39-72 years) and the male:female ratio was 2.7:1. Stereotactic and conformal RT was delivered to 5 and 34 sites, respectively. The treatment sites included the following: Bone $(n=15)$, lung $(n=9)$, brain $(n=8)$, adrenal gland $(n=2)$, renal bed $(n=2)$, skin $(n=1)$, ethmoid sinus $(n=1)$ and scalp $(n=1)$. The gap between RT and nivolumab did not exceed 2 weeks for all patients. No patients developed any grade 3-4 toxicities. Grade 2 pneumonitis was noted in 3 patients receiving lung RT. Other RT-related side effects included grade 2 lethargy $(n=2)$, grade 1 diarrhoea $(n=3)$ and grade 1 skin erythema $(n=3)$. The most common fractionation, used in $54 \%$ of the courses, was $20 \mathrm{~Gy} / 5 \mathrm{fr}$.

Response. Of the 39 treatment sites, PR and CR were noted at $25(64 \%)$ and $3(8 \%)$ sites, respectively. SD was noted at 6 sites (15\%) and PD was noted at 5 sites (13\%). Overall, $72 \%$ of the sites irradiated exhibited an excellent response to the concurrent treatment. The efficacy of RT to the bone sites was assessed by comparing the pre-treatment pain intensity 
scores using an 11-categorical point scale $(0=$ lack of pain and $10=$ worst pain imaginable) to the $6-8$-week follow-up pain score assessment. Pain response was defined as a reduction in pain scores by $\geq 2$ points compared to baseline. The median baseline pain score was 7 (range, 5-10) and pain response was observed in 9 of the 15 treatment sites $(60 \%)$. The median time spent on nivolumab from the date of the first fraction of RT was 4.5 months (range, 1.5-29 months) for patients with mNSCLC and 5 months (range, 1-38.5 months) for patients with mRCC.

\section{Discussion}

The rationale for combining $\mathrm{RT}$ and CPIs is that $\mathrm{RT}$ may lead to polyclonal T-cell infiltration and expansion at the treatment site and, in addition, CPIs block the PD-1/PD-L1 axis generating a systemic T-cell response (10). Dovedi et al (11) demonstrated the synergistic potential of RT and anti-PD-1 treatment in their study using dual tumour-bearing mouse models. In that study, it was observed that RT alone was highly effective for disease control at the irradiated site, but there were no abscopal responses. However, when RT was delivered concurrently with an anti-PD-1 monoclonal antibody, there was regression of both the irradiated and out-of-field tumours, with $>70 \%$ of mice undergoing complete responses suggesting a synergistic potential for this combination. Deng et al (12) demonstrated that the combination of RT and PD-L1 inhibitors exerted a notably more significant effect on tumour growth in mouse models compared with either treatment alone. Twyman-Saint Victor et al (13) observed marked tumour regression in a subset of patients with metastatic melanoma treated with an anti-CTLA4 antibody; they also noted that resistance was common, but could be overcome with the addition of RT and anti-PD-L1 treatment. The authors subsequently demonstrated that RT along with dual checkpoint blockade may activate non-redundant immune mechanisms and reverse $\mathrm{T}$-cell exhaustion, leading to improved efficacy. Shaverdian et al (14) assessed the disease control and pulmonary toxicity in the phase I Keynote-001 trial for patients with NSLC who previously received RT prior to pembrolizumab. Of the 98 patients who received pembrolizumab on that trial, $43 \%$ had received prior RT. There was a significant improvement in PFS $[\mathrm{HR}=0.56$ (95\% CI: 0.34-0.91), $\mathrm{P}=0.019]$ and $\mathrm{OS}$ [HR=0.58 (95\% CI: $0.36-0.94) ; \mathrm{P}=0.026$ ] for patients treated with pembrolizumab and RT compared with pembrolizumab alone, with an acceptable toxicity profile. In their retrospective study, Samstein et al (15) demonstrated an improvement in OS with concurrent CPIs and RT, particularly when CPI therapy was initiated at least 1 month prior to RT.

NIVES, a phase II multicentre trial, is the first prospective trial of nivolumab plus SBRT in mRCC. At a median follow-up of 15 months, the study showed acceptable safety, but the primary endpoint of improving response rate to $40 \%$ was not met. The objective response rate (ORR) in an intent-to-treat analysis was $17.4 \%$ (12 of 68 patients) and included one case of CR. An additional 28 patients (40.6\%) had SD. The estimated median OS was 22 months, and the 12-month survival rate was $73.4 \%$ (16).

However, a higher ORR was observed with the use of dual checkpoint inhibition in the RADVAX trial. RADVAX, a small, multi-institution, single-arm phase II study assessed the safety and efficacy of SBRT in combination with nivolumab + ipilimumab in patients with mRCC (15). Hammers et al (17) reported that the ORR was $56 \%$ during a median follow-up of 24 months for the 25 patients. SBRT was administered to 1-2 metastatic sites at a dose of $50 \mathrm{~Gy} / 5 \mathrm{fr}$ between the first and second cycles of nivolumab-ipilimumab. The median PFS was 8.21 months and the 1-year PFS rate was $36 \%$. Grade 3-4 treatment-related adverse events were noted in $36 \%$ of the patients, with radiation pneumonitis reported in 2 patients. The authors concluded that the combination was feasible and associated with an acceptable safety profile.

Despite the significant clinical benefits of combining RT with chemotherapy, however, chemoradiotherapy protocols are often associated with increased incidence and severity of side effects (18). The unique toxicity profile of immunotherapy, which is distinct from that of conventional chemotherapeutic agents, together with the technological advances in modern RT, including highly conformal, intensity-modulated and stereotactic techniques combined with high-precision image guidance, are expected to improve the tolerability of the combination and the therapeutic ratio (19). Von Reibnitz et al (20) retrospectively evaluated the toxicity profile of patients receiving immune CPIs and thoracic RT and concluded that concurrent immunotherapy and thoracic RT may be safe. The ETOP NICOLAS, is an ongoing phase II trial evaluating the safety and efficacy of nivolumab combined with radical concurrent chemoradiotherapy (66 Gy/33 fr) in stage III NSCLC (21). Interim analysis after the initial 21 patients completed at the 3 -month post-RT follow-up has demonstrated no grade $\geq 3$ pneumonitis. The results of efficacy analysis from that study are still awaited. Tables I and II outline selected ongoing clinical trials investigating the combination of nivolumab and RT in patients with mRCC or mNSCLC.

In the present retrospective study, all patients received hypofractionated RT concurrently with nivolumab. Different hypofractionation protocols were used according to the tumour site, clinical indications and technique of irradiation. The use of hypofractionated RT in this context is based on the findings of several preclinical studies, which suggested a higher immunogenic effect when higher dose per fraction is delivered $(22,23)$. For patients with mRCC or mNSCLC with PD on nivolumab therapy there are limited treatment options and no standard guidelines. Figs. 1 and 2 illustrated the case of a 62-year-old male patient with mNSCLC who was treated with nivolumab following disease progression on platinum-based chemotherapy. As the patient developed oligometastatic PD in the lung primary (Fig. 1A and B) following 8 weeks of nivolumab therapy, he was treated with palliative RT, which resulted in PR (Fig. 1C). The patient subsequently developed oligometastatic PD in the adrenal metastasis (Fig. 2A and B) and was treated with SBRT with further PR (Fig. 2C).

In the present study, the role of adding concurrent RT for patients developing oligometastatic PD on nivolumab therapy was explored, and excellent responses were demonstrated. In accordance with other retrospective studies, the results of the present study demonstrated good tolerance for the combination of nivolumab and RT, without grades 3-4 toxicities $(24,25)$. Treatment beyond progression is currently not the standard of care for patients receiving nivolumab. Radiological 
Table I. Ongoing phase I-II clinical trials investigating the combination of nivolumab and radiotherapy in patients with $\mathrm{mRCC}$ or mNSCLC.

\begin{tabular}{|c|c|c|c|c|c|c|}
\hline Clinical trials & Phase & Indication & Treatment arms & $\begin{array}{l}\text { Number } \\
\text { of patients }\end{array}$ & $\begin{array}{l}\text { Primary } \\
\text { outcome }\end{array}$ & $\begin{array}{l}\text { Expected trial } \\
\text { completion date }\end{array}$ \\
\hline NCT03044626 & II & $\begin{array}{l}\text { Metastatic } \\
\text { non-squamous } \\
\text { NSCLC }\end{array}$ & $\begin{array}{l}\text { Arm 1: Nivolumab } 240 \mathrm{mg} \\
\mathrm{q} 2 \mathrm{w}+20 \mathrm{~Gy} / 5 \text { fractions to } \\
\text { one metastatic site } \\
\text { Arm 2: Nivolumab } 240 \mathrm{mg} \mathrm{q} 2 \mathrm{w}\end{array}$ & 130 & $\begin{array}{l}\text { Objective } \\
\text { response rate }\end{array}$ & November 2021 \\
\hline NCT02696993 & $\mathrm{I} / \mathrm{II}$ & $\begin{array}{l}\text { NSCLC with } \\
\text { brain metastases }\end{array}$ & $\begin{array}{l}\text { Nivolumab + ipilimumab + whole } \\
\text { brain radiotherapy or stereotactic } \\
\text { radiosurgery }\end{array}$ & 80 & $\begin{array}{l}\text { Maximum } \\
\text { tolerated dose } \\
\text { nivolumab }\end{array}$ & December 2020 \\
\hline NCT02781506 & II & $\begin{array}{l}\text { Metastatic clear } \\
\text { cell RCC }\end{array}$ & $\begin{array}{l}\text { Nivolumab alone: IV, } 3 \mathrm{mg} / \mathrm{kg} \\
\mathrm{q} 2 \mathrm{w}+\text { stereotactic ablative } \\
\text { radiotherapy ( } 1-3 \text { fractions for } 1-3 \\
\text { lesions) }\end{array}$ & 35 & Response rate & December 2022 \\
\hline NCT03149159 & II & $\mathrm{mRCC}$ & $\begin{array}{l}\text { Nivolumab } 3 \mathrm{mg} / \mathrm{kg}+\text { ipilimumab } \\
1 \mathrm{mg} / \mathrm{kg}+30 \mathrm{~Gy} / 5 \text { fractions }\end{array}$ & & Withdrawn & \\
\hline NCT03223155 & I & $\begin{array}{l}\text { Stage } 4 \text { small } \\
\text { cell lung cancer }\end{array}$ & $\begin{array}{l}\text { Nivolumab + ipilumumab + } \\
\text { sterotactic body radiotherapy } \\
\text { concurrent vs. sequential }\end{array}$ & 80 & $\begin{array}{l}\text { Serious } \\
\text { adverse events }\end{array}$ & December 2020 \\
\hline
\end{tabular}

RCC, renal cell carcinoma; mRCC metastatic RCC; NSCLC, non-small cell lung cancer; mNSCLC, metastatic NSCLC; q2w, every 2 weeks.

Table II. Ongoing phase III clinical trials investigating the combination of nivolumab and radiotherapy.

\begin{tabular}{|c|c|c|c|c|c|}
\hline Clinical trials & Indication & Treatment arms & $\begin{array}{c}\text { Number of } \\
\text { patients }\end{array}$ & Endpoints & $\begin{array}{c}\text { Estimated } \\
\text { completion date }\end{array}$ \\
\hline NCT03349710 & $\begin{array}{l}\text { Squamous cell } \\
\text { carcinoma of the } \\
\text { head and neck }\end{array}$ & $\begin{array}{l}\text { Cohort 1: nivolumab + RT } \\
\text { vs. cetuximab + RT } \\
\text { Cohort 2: Nivolumab + cisplatin + } \\
\text { cisplatin + RT vs. cisplatin + RT }\end{array}$ & 1,046 & Event-free survival & November 2022 \\
\hline $\begin{array}{l}\text { NCT02768558 } \\
\text { (RTOG 3505) }\end{array}$ & $\begin{array}{l}\text { Stage III } \\
\text { unresectable } \\
\text { non-small cell } \\
\text { lung cancer }\end{array}$ & $\begin{array}{l}\text { Cisplatin etoposide }+60 \\
\text { Gy concurrent RT followed by } \\
\pm \text { nivolumab } 240 \mathrm{mg} \mathrm{q} 2 \mathrm{w} \text { x } 1 \text { year }\end{array}$ & 660 & $\begin{array}{l}\text { Overall and } \\
\text { progression-free survival }\end{array}$ & October 2024 \\
\hline
\end{tabular}

RT, radiotherapy; OS, overall survival; PFS, progression-free survival; q2w, every 2 weeks.

assessment of response and treatment beyond progression on immunotherapy may represent the most robust tools for assessment of response in this indication of oligometastatic PD on nivolumab $(26,27)$. In the present study, the addition of RT and continuation of nivolumab beyond progression appear to be feasible for patients with oligometastatic PD.

One of the limitations of the present study is its retrospective nature, in addition to the limited number of cases and short follow-up interval time. The heterogeneity of the included cases in terms of primary diagnosis, RT technique and fractionation also added to these limitations. A prospective study with a larger patient population and adequate follow-up is required to further elucidate these issues. However, clinical discretion should be used in the meantime while recommending this combination of nivolumab and RT, keeping in view the emerging data and the potential benefits.

In conclusion, the results achieved with the combination of nivolumab and RT appear promising in several retrospective studies. In the present study for patients with mRCC or mNSCLC with oligometastatic PD during nivolumab therapy, the addition of RT appears to initiate a response and prolong the time on nivolumab treatment. The combination of nivolumab and RT appears to be well-tolerated, with response rates exceeding published studies of nivolumab monotherapy. 

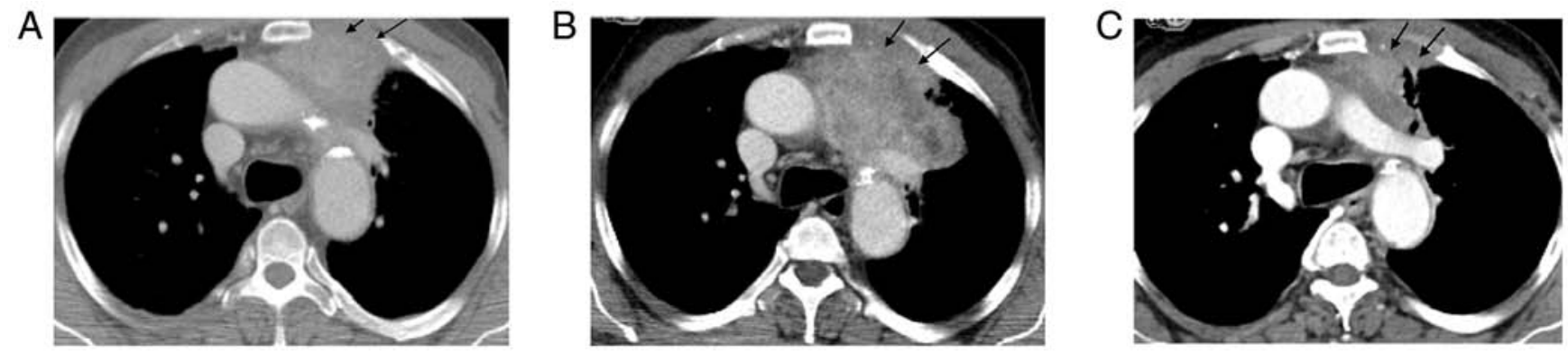

Figure 1. Imaging findings in a 62-year-old male patient with metastatic non-small cell lung cancer who was treated with second-line nivolumab and developed oligometastatic disease progression. Axial contrast-enhanced CT scan of the chest showing a left lung primary tumour (black arrows) at (A) baseline; (B) 8 weeks post-nivolumab therapy demonstrating disease progression; and (C) 8 -weeks post-radiotherapy (20 Gy in 5 fractions) showing treatment response.

A

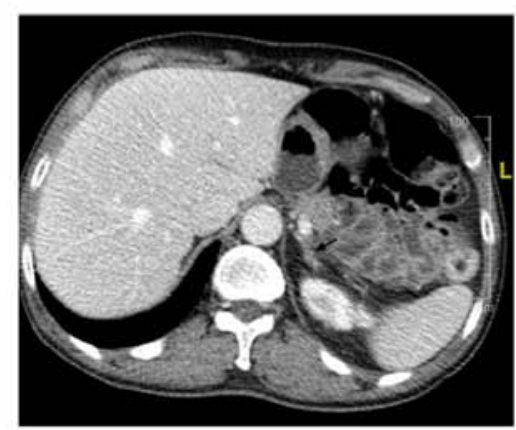

B

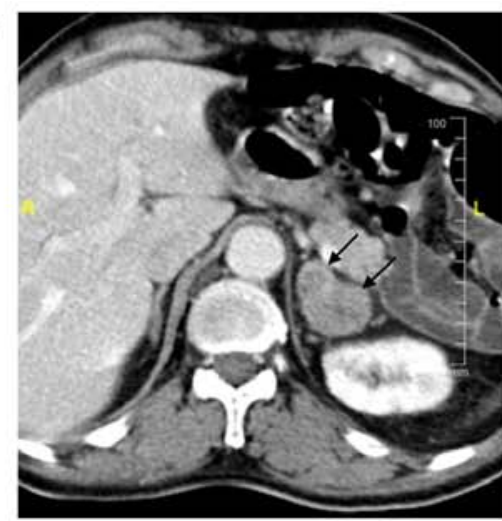

C

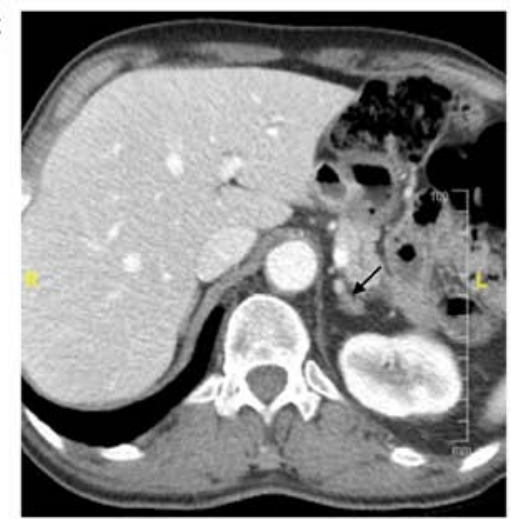

Figure 2. Imaging findings in a 62-year-old male patient with metastatic non-small cell lung cancer who was treated with second-line nivolumab and developed oligometastatic disease progression. Axial contrast-enhanced CT scan of the abdomen demonstrating a left adrenal gland metastasis (black arrows) at (A) baseline; (B) 6 months post-nivolumab therapy demonstrating disease progression; and (C) 8 weeks post-stereotactic body radiotherapy (40 Gy in 4 fractions) showing a significant reduction in the size of the left adrenal gland metastasis.

The optimal strategy for integrate RT into the immune treatment pathway still remains to be determined. Several questions regarding optimal RT dose and fractionation, treatment technique, timing and safety still remain unanswered. A plethora of clinical trials are currently investigating these RT-immune interactions (Tables I and II), however, several challenges remain to be addressed to maximize the efficacy of this promising combination.

\section{Acknowledgements}

Not applicable.

\section{Funding}

No funding was received.

\section{Availability of data and materials}

All the data relevant to the present study are available from the corresponding author on reasonable request.

\section{Authors' contributions}

JA: Primary author, conception and design, preparation and review of the manuscript; AF: Literature review, patient follow-up, preparation and review of the manuscript; AMA and MAl: Literature review and data collection; EM, AA and MAb: Case analysis, preparation and critical review of the manuscript; HA: Literature review, data interpretation, review of imaging and response assessment; $\mathrm{SH}$ and JG: Expert opinion for management of toxicities, case review, radiotherapy guidance, literature review, conception, supervision and review of the manuscript. JA and AF have seen the raw data and can confirm their authenticity. All the authors have read and approved the final manuscript.

\section{Ethics approval and consent to participate}

Ethics approval has been obtained from the Institutional Review Board of the Prince Sultan Military Medical City HP-01-R079, approval no. 1490 on 18/03/2021.

\section{Patient consent for publication}

Not applicable.

\section{Competing interests}

Dr Jawaher Ansari, Dr Abdulaziz Alhamad and Professor Syed Hussain have received lecture fees from Bristol Myers Squibb. All the other authors declare that they have no competing interests. 


\section{References}

1. Brahmer JR, Drake CG, Wollner I, Powderly JD, Picus J, Sharfman WH, Stankevich E, Pons A, Salay TM, McMiller TL, et al: Phase I study of single-agent anti-programmed death-1 (MDX-1106) in refractory solid tumors: Safety, clinical activity, pharmacodynamics, and immunologic correlates. J Clin Oncol 28: 3167-3175, 2010.

2. Gandhi L, Rodríguez-Abreu D, Gadgeel S, Esteban E, Felip E, De Angelis F, Domine M, Clingan P, Hochmair MJ, Powell SF, et al: KEYNOTE-189 investigators: Pembrolizumab plus chemotherapy in metastatic non-small-cell lung cancer. N Engl J Med 378: 2078-2092, 2018.

3. Larkin J, Chiarion-Sileni V, Gonzalez R, Grob JJ, Cowey CL, Lao CD, Schadendorf D, Dummer R, Smylie M, Rutkowski $\mathrm{P}$, et al: Combined nivolumab and ipilimumab or monotherapy in untreated melanoma. N Engl J Med 373: 23-34, 2015.

4. Delaney G, Jacob S, Featherstone C and Barton M: The role of radiotherapy in cancer treatment: Estimating optimal utilization from a review of evidence-based clinical guidelines. Cancer 104: $1129-1137,2005$

5. Ringborg U, Bergqvist D, Brorsson B, Cavallin-Ståhl E, Ceberg J, Einhorn N, Frödin JE, Järhult J, Lamnevik G, Lindholm C, et al: The swedish council on technology assessment in health care (SBU) systematic overview of radiotherapy for cancer including a prospective survey of radiotherapy practice in Sweden 2001-summary and conclusions. Acta Oncol 42: 357-365, 2003.

6. Walle T, Martinez Monge R, Cerwenka A, Ajona D, Melero I and Lecanda F: Radiation effects on antitumor immune responses: Current perspectives and challenges. Ther Adv Med Oncol 10: 1758834017742575,2018

7. Demaria S, Ng B, Devitt ML, Babb JS, Kawashima N, Liebes L and Formenti SC: Ionizing radiation inhibition of distant untreated tumors (abscopal effect) is immune mediated. Int J Radiat Oncol Biol Phys 58: 862-870, 2004.

8. Eisenhauer EA, Therasse P, Bogaerts J, Schwartz LH, Sargent D, Ford R, Dancey J, Arbuck S, Gwyther S, Mooney M, et al: New response evaluation criteria in solid tumours: Revised RECIST guideline (version 1.1). Eur J Cancer 45: 228-247, 2009.

9. Trotti A, Colevas AD, Setser A and Basch E: Patient-reported outcomes and the evolution of adverse event reporting in oncology. J Clin Oncol 25: 5121-5127, 2007.

10. Colton M, Cheadle EJ, Honeychurch J and Illidge TM: Reprogramming the tumour microenvironment by radiotherapy: Implications for radiotherapy and immunotherapy combinations. Radiat Oncol 15: 254, 2020.

11. Dovedi SJ, Cheadle EJ, Popple AL, Poon E, Morrow M, Stewart R, Yusko EC, Sanders CM, Vignali M, Emerson RO, et al: Fractionated radiation therapy stimulates antitumor immunity mediated by both resident and infiltrating polyclonal T-cell populations when combined with PD-1 blockade. Clin Cancer Res 23: 5514-5526, 2017.

12. Deng L, Liang H, Burnette B, Beckett M, Darga T, Weichselbaum RR and Fu YX: Irradiation and anti-PD-L1 treatment synergistically promote antitumor immunity in mice. J Clin Invest 124: 687-695, 2014.

13. Twyman-Saint Victor C, Rech AJ, Maity A, Rengan R,Pauken KE, Stelekati E, Benci JL, Xu B, Dada H, Odorizzi PM, et al: Radiation and dual checkpoint blockade activate non-redundant immune mechanisms in cancer. Nature 520: 373-377, 2015.

14. Shaverdian N, Lisberg AE, Bornazyan K, Veruttipong D, Goldman JW, Formenti SC, Garon EB and Lee P: Previous radiotherapy and the clinical activity and toxicity of pembrolizumab in the treatment of non-small-cell lung cancer: A secondary analysis of the KEYNOTE-001 phase 1 trial. Lancet Oncol 18 : 895-903, 2017
15. Samstein R, Rimner A, Barker CA and Yamada Y: Combined immune checkpoint blockade and radiation therapy: timing and dose fractionation associated with greatest survival duration among over 750 treated patients. Int J Radiat Oncol Biol Phys 99 (Suppl 2): S129-S130, 2017.

16. Masini C, Iotti C, De Giorgi U, Bellia RS, Buti S, Salaroli F, Zampiva I, Mazzarotto R, Mucciarini C, Baldessari C, et al: Nivolumab (NIVO) in combination with stereotactic body radiotherapy (SBRT) in pretreated patients (pts) with metastatic renal cell carcinoma (mRCC): First results of phase II NIVES study. J Clin Oncol 38 (6 Suppl): S613, 2020.

17. Hammers HJ, Vonmerveldt D, Ahn C, Nadal RM, Drake CG, Folkert MR, Lain AM, Courtney KD, Brugarolas J, Song DY, et al: Combination of dual immune checkpoint inhibition (ICI) with stereotactic radiation (SBRT) in metastatic renal cell carcinoma (mRCC) (RADVAX RCC). J Clin Oncol 38 (Suppl 6): 614-614, 2020.

18. Deutsch E, Chargari C, Galluzzi L and Kroemer G: Optimising efficacy and reducing toxicity of anticancer radioimmunotherapy. Lancet Oncol 20: e452-e463, 2019.

19. Jagodinsky JC, Harari PM and Morris ZS: The promise of combining radiation therapy with immunotherapy. Int J Radiat Oncol Biol Phys 108: 6-16, 2020.

20. Von Reibnitz D, Chaft JE, Wu AJ, Samstein R, Hellmann MD, Plodkowski AJ, Zhang Z, Shi W, Dick-Godfrey R, Panchoo KH, et al: Safety of combining thoracic radiation therapy with concurrent versus sequential immune checkpoint inhibition. Adv Radiat Oncol 3: 391-398, 2018.

21. Peters S, Felip E, Dafni U, Belka C, Guckenberger M, Irigoyen A, Nadal E, Becker A, Vees H, Pless M, et al: Safety evaluation of nivolumab added concurrently to radiotherapy in a standard first line chemo-radiotherapy regimen in stage III non-small cell lung cancer-The ETOP NICOLAS trial. Lung Cancer 133: 83-87, 2019.

22. Chajon E, Castelli J, Marsiglia H and De Crevoisier R: The synergistic effect of radiotherapy and immunotherapy: A promising but not simple partnership. Crit Rev Oncol Hematol 111: 124-132, 2017

23. Camphausen K, Moses MA, Ménard C, Sproull M, Beecken WD, Folkman J and O'Reilly MS: Radiation abscopal antitumor effect is mediated through p53. Cancer Res 63: 1990-1993, 2003.

24. Amin NP, Zainib M, Parker SM, Agarwal M and Mattes MD: Multicenter report on toxicities of concurrent nivolumab and radiation therapy. Adv Radiat Oncol 3: 399-404, 2018.

25. Bang A, Wilhite TJ, Pike LRG, Cagney DN, Aizer AA, Taylor A, Spektor A, Krishnan M, Ott PA, Balboni TA, et al: Multicenter evaluation of the tolerability of combined treatment with PD-1 and CTLA-4 immune checkpoint inhibitors and palliative radiation therapy. Int J Radiat Oncol Biol Phys 98: 344-351, 2017.

26. Dromain C, Beigelman C, Pozzessere C, Duran R and Digklia A: Imaging of tumour response to immunotherapy. Eur Radiol Exp 4: 2, 2020.

27. Ge X, Zhang Z, Zhang S, Yuan F, Zhang F, Yan X, Han X, Ma J, Wang L, Tao H, et al: Immunotherapy beyond progression in patients with advanced non-small cell lung cancer. Transl Lung Cancer Res 9: 2391-2400, 2020.

This work is licensed under a Creative Commons Attribution-NonCommercial-NoDerivatives 4.0 International (CC BY-NC-ND 4.0) License. 